\title{
Las representaciones y prácticas docentes acerca de los materiales curriculares. Cambios y permanencias*
}

\section{Teachers' Practices and Representations on Curricular Materials. Changes and Continuities}

\author{
Antonio Soto Rosales ${ }^{a}$ \\ Universidad de Huelva, España \\ ORCID: http://orcid.org/0000-0003-3752-5970 \\ Gabriel Travé GonZÁlez \\ Universidad de Huelva, España \\ ORCID: http://orcid.org/0000-0002-1339-9239
}

a Autor de correspondencia: Correo electrónico: soto@uhu.es

Para citar este artículo: Soto Rosales, A., \& Travé González, G. (2018). Las representaciones y prácticas docentes acerca de los materiales curriculares. Cambios y permanencias. Universitas Psychologica 17(5), 1-11. http://doi.org/10.11144/Javeriana.upsy17 $-5 . \mathrm{rpdm}$

\section{RESUMEN}

Se presentan los resultados de una investigación interesada en las concepciones que mantiene el profesorado sobre la utilización de materiales curriculares en la enseñanza de Conocimiento del Medio. A partir de un cuestionario construido para tal efecto, se obtuvo información de 863 profesores de las ocho provincias de Andalucía (España). El análisis de correspondencias múltiples efectuado revela la existencia de tres factores que explican el $71.2 \%$ de la varianza: el primero representa las ideas propias del idealismo novel frente al realismo que genera la experiencia; el segundo, las ideas de defensa a ultranza de la utilidad del libro de texto frente a la duda sobre su utilidad y un tercero de carácter metodológico enfrenta metodologías activas a metodologías tradicionales. Palabras clave

concepciones del profesorado; materiales curriculares; libros de texto; materiales de elaboración docente; análisis de correspondencias múltiples.

\footnotetext{
ABSTRACT

This work presents the results of an investigation into the conceptions teachers hold on the use of curricular materials in the teaching of Natural and Social Science. Based on a questionnaire built for this purpose, information from 863 teachers from the eight provinces of Andalusia (Spain) was obtained. The analysis of multiple correspondences, reveals the existence of three factors that explain $71.2 \%$ of the variance, the first represents the ideas linked to beginners' idealism versus the realism generated by experience; the second, the support of the textbook versus the doubt about its usefulness, and a third, the active methodologies versus more traditional approaches.

Keywords

teacher knowledge; instructional materials; textbooks; teacher developed materials; multiple correspondence analysis.
} 


\section{Introducción}

La abundante producción de la investigación referida al análisis de los manuales escolares y los materiales de elaboración docente utilizados por el profesorado (Rodríguez \& Álvarez, 2017; Schizas, Papatheodorou, \& Stamou, 2018) contrasta paradójicamente con un examen que requiere mayor atención científica. Los estudios relativos a las concepciones y las prácticas docentes sobre la utilización de los materiales curriculares continúan siendo escasos, a pesar de la importancia que entrañan sus resultados para el cambio educativo (Chingos \& Whitehurst, 2012).

Los trabajos desarrollados en este campo de investigación didáctica constatan la relación existente entre las concepciones profesionales del profesorado y el uso que hacen de los materiales curriculares. Esta concordancia depende indudablemente del modelo didáctico docente (Pilitsis \& Duncan 2012). En este sentido, sostienen Solís, Porlán, Rivero y Martín (2012) la existencia de cuatro modelos didácticos que utilizan diversas tipologías de materiales curriculares: el modelo tradicional emplea exclusivamente libros de texto, el modelo tecnológico usa fichas y manuales escolares, el modelo espontaneísta desarrolla en la práctica fichas de materiales de elaboración docente y, por último, el modelo de investigación en la escuela elabora e implementa diversas estrategias didácticas, entre otras, proyectos de trabajo, propuestas de investigación escolar o de investigación en el medio. Asimismo, Cañal, Travé y Pozuelos (2011) constatan que estos modelos están íntimamente relacionados con el nivel de desarrollo profesional alcanzado, y afirman que los docentes que diseñan y desarrollan materiales de elaboración propia en su práctica de aula, están convencidos de la enseñanza por indagación, mientras que los que dependen exclusivamente del libro de texto continúan persuadidos de las ventajas del texto escolar como material hegemónico para su uso en clase.

La trascendencia que posee esta línea de investigación, centrada en el análisis del pensamiento y la práctica docente de los materiales curriculares, trata de encontrar soluciones, entre otras, a dos paradojas que condicionan la práctica docente. En primer lugar, la mayor parte de los estudios sobre libros de texto asegura que configuran el material mayoritario en las escuelas actuales (Pingel, 2010), sin embargo, otros trabajos señalan que más de la mitad del profesorado considera que los textos escolares generan aprendizajes memorísticos y obvian el desarrollo de las competencias (Travé, Pozuelos, $\&$ Soto, 2015). En el mismo sentido, los estudios de Abdel Latif (2012), demuestran que el uso de los libros de texto basados en el logro de estándares favorece el cambio en la práctica docente; los resultados indican que este tipo de reformas curriculares no ha producido los cambios deseados en las prácticas docentes, señalando las carencias inherentes a su uso.

En segundo lugar, la tradición innovadora de la educación (Stenhouse, 1997), basada en el protagonismo del profesorado que diseña, desarrolla y evalúa los materiales curriculares, ha ido perdiendo peso con el paso del tiempo; mientras que curiosamente diversos estudios revelan que un sector destacado del colectivo docente señala que esta práctica de aula representa la esencia del cambio educativo. En esta línea, Polat (2010), indagando acerca de la percepción generada por el uso de materiales elaborados por colectivos docentes, sugiere que los participantes mejoran sus creencias sobre la eficacia que estos materiales poseen para incrementar la calidad de la enseñanza y el desarrollo profesional. Por su parte, Mansour (2010) analiza la relación existente entre las concepciones de los profesores y el modo de desarrollar sus prácticas, constatando la necesidad de considerar las creencias y los conocimientos docentes, así como los factores socioculturales, por su influencia en la práctica educativa. Sin embargo, no debemos olvidar, por último, que el colectivo docente adolece de conocimientos y destrezas necesarias para el diseño de materiales, debido a las carencias detectadas en la formación inicial y permanente del profesorado (Huizinga, Handelzalts, Nieveen, 
$\&$ Voogt, 2014). Este es el contexto en el que se inserta el presente trabajo.

\section{Método}

Este informe forma parte de un estudio más extenso en el que se ha intentado integrar metodologías cualitativas y cuantitativas en aras de lograr una mayor comprensión de las cuestiones en estudio. Los objetivos perseguidos fueron: de una parte, analizar e interpretar las concepciones y prácticas docentes acerca de la utilización de los libros de texto y de los materiales de elaboración propia, y de otra, establecer a través de un análisis de correspondencias múltiples las ideas fundamentales que subyacen al cuestionario.

Para la obtención de los datos, se elaboró un cuestionario ad hoc compuesto por 66 ítems; la primera parte, compuesta por un total de 15 ítems de carácter ilustrativo con los que se obtuvo información sobre datos personales, académicos y profesionales de los participantes, y la segunda parte (51 ítems, formato escala de Likert con cuatro opciones de respuesta) constituida por dos categorías: una primera para indagar sobre la concepciones del profesorado sobre el libro de texto y otros materiales curriculares y la segunda interesada en las concepciones del profesorado sobre su práctica con los materiales curriculares. El cuestionario fue validado a través del juicio de expertos (siete profesores de distintas universidades y ocho maestros). El índice de fiabilidad obtenido a través del coeficiente Spearman-Brown fue de 0.787.

A los encuestados se les permitió añadir cuantos comentarios aclaraciones y matices consideraron oportunos en relación con cada uno de los ítems, en un espacio dispuesto para tal efecto. La selección de la muestra se realizó en las ocho provincias andaluzas, a través de muestreo por conglomerados geográficos; como criterios añadidos también se tuvieron en cuenta otras variables tales como ubicación (capital o provincia), comarca y tamaño del centro educativo. Los 863 cuestionarios obtenidos, adecuadamente cumplimentados, suponen asumir un margen de error del $5 \%$ a un nivel de confianza del $99 \%$. Se logró información de 94 centros de Educación Infantil y Primaria de 81 localidades andaluzas.

\section{Características de la muestra}

Las características más representativas de nuestra muestra fueron: edad media de 43 años; el intervalo más numeroso, entre 31 a 39 años, representó el $20.7 \%$; el $74.9 \%$ correspondió a mujeres y el $25.1 \%$ a varones; la titularidad mayoritaria fue de centros públicos (95.9\%), con un $4.1 \%$ de centros concertados. El 77.9 $\%$ del profesorado impartía el nivel educativo de Educación Primaria, frente al $22.1 \%$ de Educación Infantil. El número de años de experiencia docente fue de 19.9 años de media y el rango de edad mayoritario (25.8 \%) estaba comprendido entre los 21 y los 30 años. En el caso de experiencia docente en Conocimiento de Medio, la media resultó de 17.4 años; el número medio de alumnos por clase fue de 22.59. Algo más de la mitad del profesorado de la muestra desarrollaba funciones de tutoría $(54.7 \%)$ y el $17.7 \%$, la coordinación de ciclo. Las editoriales más usadas en los contextos académicos fueron Anaya 29 \%, Santillana 20.7 \%, SM $10.7 \%$, Edelvives $5.1 \%$, Everest $3.4 \%$ y Vicens Vives $3 \%$. Según los datos obtenidos, el $22.2 \%$ afirmó que diseñaba unidades didácticas y fichas, mientras que solo el $5.4 \%$ trabajaba con unidades didácticas publicadas por la Consejería de Educación.

\section{Resultados}

\section{Análisis de correspondencias múltiples}

La multidimensionalidad de la escala hizo aconsejable usar algún tipo de estrategia que permitiera reducir y explicitar sus dimensiones. El abordaje estadístico acorde con esta premisa fue el análisis de correspondencias múltiples (en adelante ACM). También se incluyeron los resultados del análisis de clúster efectuado. 
La matriz de datos factoriales que muestra el programa utilizado (SPAD V3.5) (Lebart, Morineau, \& Warwick, 1984) presenta los ítems contenidos en cada factor de forma dicotómica, ordenando en cada polo los interdependientes en función de su peso en la configuración del factor (V-Test), que será siempre positivo en un extremo y negativo en el otro.

A continuación, en la Tabla 1 se presentan los estadísticos relativos a los cinco primeros factores encontrados, con expresión de los porcentajes de varianza explicados por cada uno de ellos.

\section{Tabla 1}

Histograma correspondiente al análisis de correspondencias múltiples

\begin{tabular}{lrrr}
\hline $\mathrm{N}^{\circ}$ & Valor propio & \% Varianza explicada & Histograma \\
\hline 1 & 0.15 & 34.125 & $* * * * * * * * * * * * * * * * * * * * * * * * * * * * * * * * * * *$ \\
2 & 0.127 & 22.02 & $* * * * * * * * * * * * * * * * * * * * * *$ \\
3 & 0.075 & 15.055 & $* * * * * * * * * * * * * *$ \\
4 & 0.005 & 1.703 & $* *$ \\
5 & 0.004 & 1.326 & $*$ \\
\hline
\end{tabular}

A partir de los datos se ha considerado suficiente el análisis de los tres primeros factores, que explican el $71.2 \%$ de la varianza. El salto cuantitativo de los siguientes factores es lo suficientemente amplio como para prescindir de ellos. Es necesario mencionar que el "valor propio" que ofrece el programa (SPAD) debe traducirse a porcentaje de varianza, para lo cual se utilizó el procedimiento propuesto por Benzécri (1976).

\section{Tabla 2}

Ítems agrupados en el Factor 1/3

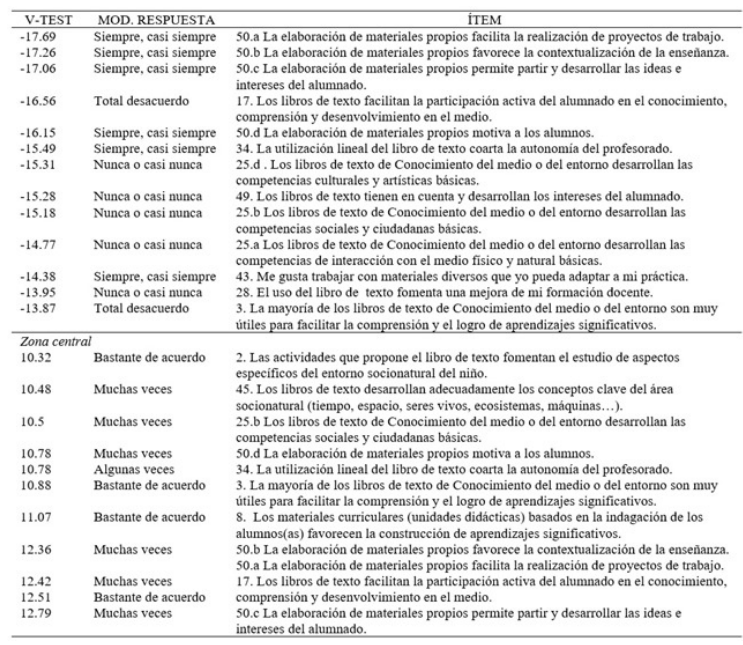

Como se observa en la Tabla 2, el análisis de este primer eje del ACM manifiesta, en su polo negativo, modalidades de respuesta siempre extremas en relación con dos ejes: ventajas de la elaboración propia de los materiales curriculares y desventajas de los libros de texto. En relación con las ventajas, los ítems más representativos en la configuración del factor plantean que la elaboración de materiales propios facilita, entre otros, la realización de proyectos de trabajo, contextualiza la enseñanza, permite desarrollar ideas e intereses de los alumnos, motiva a los alumnos, aumenta el desarrollo profesional de los enseñantes, procura satisfacción a los profesionales, favorece la construcción de aprendizajes significativos. Por el contrario, entre las desventajas del uso del libro de texto, hay que anotar que no facilita la participación activa de los alumnos, coarta la autonomía del profesorado, no desarrolla competencias básicas a través de la realización de actividades prácticas, no fomenta la formación docente, no es útil para el logro de aprendizajes significativos, así como no desarrolla adecuadamente los conceptos clave del área sociocultural. En el polo positivo, se repite buena parte de las ventajas del uso de los materiales curriculares de elaboración propia, aunque con opciones de respuesta más moderadas y, en un sentido marcadamente contrario al polo negativo, se proponen ventajas al uso del libro de texto, también con opciones de respuesta moderada: facilita la participación activa del alumnado, es útil para el logro de aprendizajes significativos y desarrolla competencias básicas.

Las modalidades ilustrativas señalan que el polo negativo correlaciona con la especialidad de Educación infantil, así como con las edades comprendidas entre los 26 y los 30 años, por el contrario, el polo positivo mantiene correlaciones con los maestros de educación primaria de edades comprendidas entre los 50 y 60 años. Parece que en este eje se confrontan las ideas propias del idealismo novel frente al realismo que genera la experiencia. 
Tabla 3

Ítems agrupados en Factor 2/3

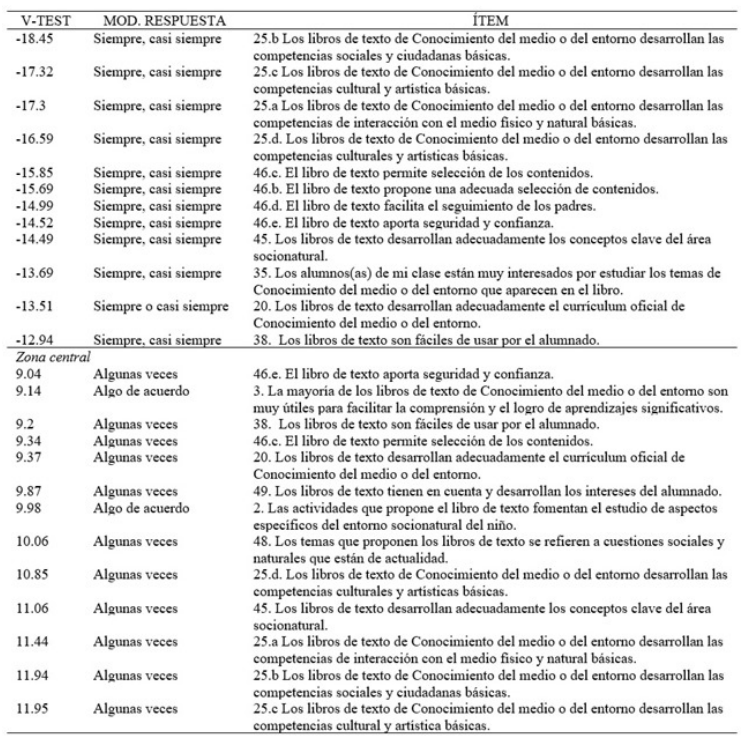

El polo negativo de este segundo factor aglutina una serie de ítems que apuntan hacia una valoración positiva del uso de los libros de texto como material curricular esencial en clase (ver Tabla 3), así, por ejemplo, se propone que los libros de texto desarrollan las competencias básicas de los alumnos, ofrece ventajas tales como ahorrar tiempo, permite la selección de contenidos, aporta seguridad; los textos desarrollan adecuadamente los conceptos clave del área sociocultural; interesan los alumnos, etc. Las opciones de respuesta que se pueden apreciar en este polo son siempre extremas (siempre o casi siempre).

El polo positivo supone una repetición de lo que se acaba de describir en el polo negativo con una diferencia que se centra en las opciones de respuesta asociadas, que en este caso son mucho más moderadas (algunas veces, algo de acuerdo), lo que sitúa este polo en una tendencia que resulta prácticamente desfavorable al uso de los libros de texto como materiales curriculares adecuados para las clases.

Las variables ilustrativas muestran que el polo negativo correlaciona con la especialidad de Educación Infantil y con las edades comprendidas entre 56 y 60 años y el polo positivo con aquellos maestros que además tienen alguna licenciatura cuyas edades están comprendidas entre 31 y 35 años. Por lo anterior, parece que este eje enfrenta a la defensa a ultranza de la utilidad del libro de texto (polo negativo) con la duda invariada sobre su utilidad.

\section{Tabla 4}

Ítems agrupados Factor 3/3

\begin{tabular}{|c|c|c|}
\hline V-TEST & MOD. RESPUESTA & ITEM \\
\hline-11.72 & Muchas veces & $\begin{array}{l}\text { 31.c. Realizo experiencias y prácticas para trabajar conocimiento del medio o del } \\
\text { entomo. }\end{array}$ \\
\hline-11.71 & Muchas veces & 47. Utilizo en mis clases materiales curriculares que favorecen la indagación y la \\
\hline-9.22 & Muchas veces & 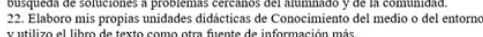 \\
\hline .8 .89 & Siempre, casi siempre & 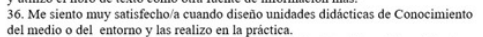 \\
\hline-8.46 & $\begin{array}{l}\text { Muchas veces } \\
\text { Siempre, casi siempre }\end{array}$ & 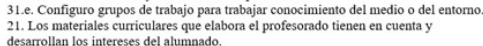 \\
\hline & Algunas veces & $\begin{array}{l}\text { 40. El material fundamental que utilizo en clase es el libro de texto. } \\
\text { theso }\end{array}$ \\
\hline & Siempre, casi siempre & 31.d. Realizo debates para trabajar conocimiento del medio o del entorno. \\
\hline & Siempre, casi s & 43. Me gusta trabajar con materiales diversos que yo pueda adaptar a mi práctica. \\
\hline & Muchas veces & $\begin{array}{l}\text { 30. El uso de unidades didácticas y otros materiales curriculares me permite indagar } \\
\text { con mis alumnos(as) cuestiones sobre el medio. }\end{array}$ \\
\hline-6.87 & Siempre, casi siempre & 50.d. La elaboración de materiales propios motiva a los alumnos. \\
\hline-6.67 & Siempre, casi siempre & $\begin{array}{l}\text { 50.c La elaboración de materiales propios permite partir y desarrollar las ideas } \mathrm{e} \\
\text { intereses del alumnado. }\end{array}$ \\
\hline \multicolumn{3}{|r|}{. } \\
\hline & & \\
\hline 8.51 & $\begin{array}{l}\text { Agumas veces } \\
\text { Siempre, casi s }\end{array}$ & $\begin{array}{l}\text { 40. Realizo la calificación de los alumnos-as a través de los exámenes proporcionados } \\
\text { 4lo rel libro del masestro. }\end{array}$ \\
\hline 8.72 & Siempre, casi siempre & $\begin{array}{l}\text { 23. La evaluación del alumnado la realizo a través de una prueba oral o escrita de los } \\
\end{array}$ \\
\hline 9.02 & Algunas veces & 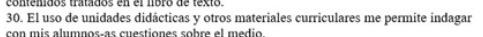 \\
\hline 9.33 & Algunas veces & 32. Evaluo a mis alumnos (as) mediante la participación y los trabajos individuales $y$ \\
\hline 9.49 & Nunca o casi nunca & $\begin{array}{l}\text { 31.e. Configuro grupos de trabajo par. } \\
\text { 3. }\end{array}$ \\
\hline $\begin{array}{l}10.79 \\
11.33\end{array}$ & $\begin{array}{l}\text { Aggunas veces } \\
\text { Nunca o casi nunca }\end{array}$ & $\begin{array}{l}\text { 43. Me gusta trabajar con materiales diversos que yo pueda adaptar a mi práctica. } \\
\text { 31.c. Realizo experiencias y prácticas para trabajara conocimiento del medio o del }\end{array}$ \\
\hline & & \\
\hline $\begin{array}{lll}11.78 \\
11.72\end{array}$ & $\begin{array}{l}\text { Siempre, cass sil } \\
\text { Nunca o casi nus }\end{array}$ & $\begin{array}{l}\text { 47. Utililo en mis clases materiales surriculares que favorecen la indagación y la } \\
\text { busqueda de soluciones a problemas cercanos del alumnado yde la comunidad.| }\end{array}$ \\
\hline 13.14 & Nunca o casi nunca & 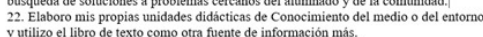 \\
\hline
\end{tabular}

Los ítems que figuran en el polo negativo de este tercer factor (Tabla 4) ofrecen la siguiente información: para trabajar conocimiento del medio, se realizan actividades prácticas tales como salidas al entorno, experiencias prácticas, debates, grupos de trabajo, etc.; se usan materiales curriculares que favorecen la indagación y la búsqueda de soluciones a problemas cercanos al alumno; se asume la mejora en la satisfacción profesional cuando se diseñan personalmente las unidades didácticas de conocimiento del medio; los materiales curriculares elaborados por el profesorado tienen en cuenta y desarrollan los intereses del alumnado; favorecen la motivación de los alumnos; utilizan el medio donde se ubica la escuela y, en la evaluación, no se suelen usar pruebas orales o escritas basadas en contenidos de libros de texto, etc. El polo positivo aglutina prácticamente los mismos ítems con una diferencia clara en las opciones de respuesta elegidas, que en este caso se oponen a los contenidos que se mencionaron con anterioridad. Las modalidades ilustrativas señalan que el polo negativo correlaciona con la especialidad de Educación infantil, con las 
edades comprendidas entre 26 y 30 años y ser mujer, por el contrario, el polo positivo mantiene correlación con ser hombre. Es por ello que se trata de un factor de carácter marcadamente metodológico que enfrenta a las metodologías más activas (polo negativo) con metodologías más tradicionales (polo positivo).

\section{Análisis de clúster}

Aunque no es el objetivo central, se consideró oportuno complementar los datos que se acaban de exponer con un análisis de clúster, con el objetivo de clasificar a los sujetos en distintos grupos en función de la semejanza de sus respuestas al cuestionario y afianzar las interpretaciones. Para decidir el número total de clústeres por elegir, resulta de mucha ayuda la visión gráfica del dendograma que genera el programa. En el presente caso, es prácticamente obligado decantarse por realizar cuatro agrupaciones, como se puede observar en la Figura 1.

\section{Figura 1}

Dendograma de clústeres en porcentaje

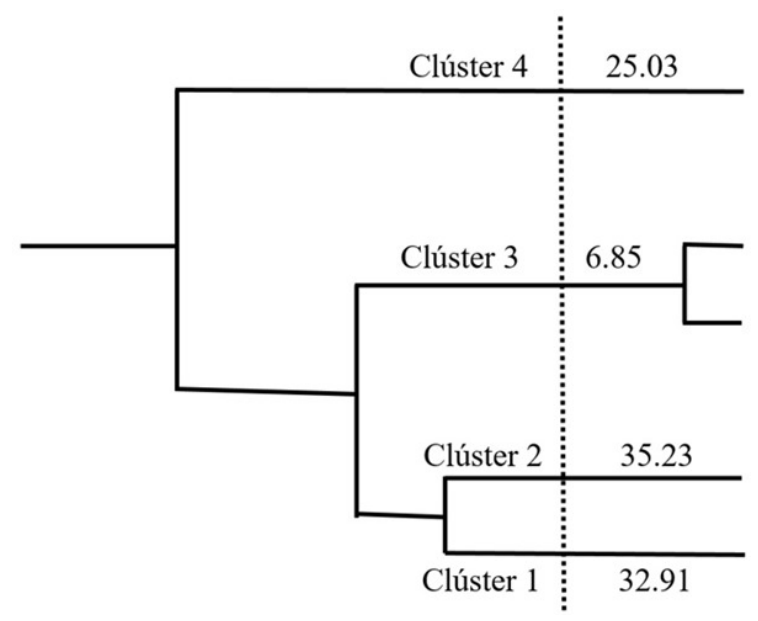

\section{Clúster 1. Profesorado funcional}

El primer clúster, que representa el $32.91 \%$ de la muestra, agrupa profesores que ensalzan las numerosas ventajas del uso del libro de texto, sin añadir comentarios contrarios a la elaboración personal de materiales curriculares. Se alejan siempre de las opciones de respuesta extremas, prefiriendo decantarse siempre por las intermedias, es por ello que el clúster parece quedar conformado por un grupo de profesores "favorables al libro de texto de carácter moderado". El 38.5 \% del clúster está conformado por maestros de Educación Primaria y el $23 \%$ de Educación Infantil; aproximadamente, la mitad se sitúan entre los 56 y los 60 años de edad.

\section{Clúster 2. Profesorado ecléctico}

La lectura de los ítems agrupados en este clúster, hace suponer que este está conformado por un grupo de profesores eminentemente prácticos, que usan los libros de texto y defienden sus ventajas y, a la par, también los beneficios que supone la elaboración de materiales propios especialmente adaptados. Las opciones de respuesta elegidas señalan siempre moderación, en la doble defensa que manifiestan. La clave interpretativa de este grupo, tal vez resida en uno de los ítems aquí agrupados, en el que manifiestan que "les gusta trabajar con materiales diversos que puedan adaptar a su práctica". Parecen ser profesores que usan todo aquello que pueda serles útil, independientemente de su procedencia. El 44.13 $\%$ del profesorado entre 31 y 35 años de edad (experiencia intermedia) han quedado incluidos en este clúster.

\section{Clúster 3. Profesorado dependiente}

Representa solo el $6.85 \%$ de la muestra, aunque su idiosincrasia resulta muy significativa. El clúster se presenta muy homogéneo tanto en los ítems que agrupa como en las opciones de respuesta elegidas. Entre sus características, apuestan de forma contundente por las bondades emanadas de los libros de texto. Las opciones de respuesta elegidas, para destacar estas bondades, son siempre extremas, manifestando así su militancia con lo que aseveran. A partir de estas características, se podría denominar al grupo 
como "pro libros de texto de carácter extremo". El $20 \%$ del profesorado entre 56 y 60 años pertenece al clúster.

\section{Clúster 4. Profesorado diseñador}

Este clúster supone la réplica del anterior por cuanto supone asumir de forma incondicional y categórica las excelencias de la elaboración de materiales propios y el rechazo del uso de los libros de texto. Se trata de un grupo de "extremos defensores de la elaboración de materiales propios y críticos con el uso de libros de texto". El $38.64 \%$ de los sujetos que quedan aquí agrupados tienen entre 26 y 30 años.

\section{Figura 2}

Proyección clústeres en Factores 1 y 2

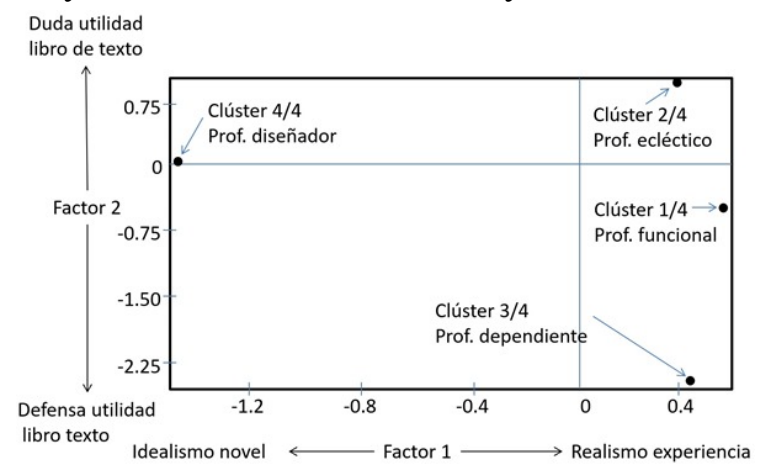

Figura 3

Proyección de clústeres en Factores 1 y 3

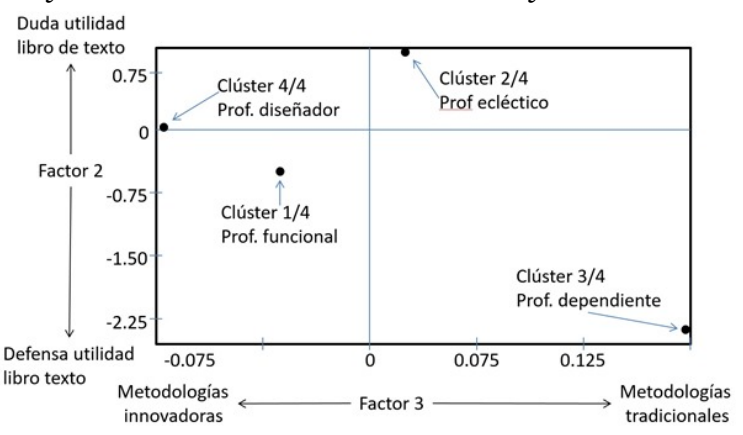

\section{Discusión}

El análisis de correspondencias efectuado, objetivo prioritario de este trabajo, informa que la composición básica del cuestionario empleado gira en torno a tres factores que explican el
$71.2 \%$ de su varianza. El primero de ellos (34.125\% de la varianza), sitúa en su polo negativo aquellos ítems que proponen, en forma extrema, las ventajas en la elaboración personal de materiales curriculares, y a la vez plantean las desventajas en el uso de los libros de texto. En su polo positivo, se agrupan los ítems que, de forma moderada, señalan las ventajas de la elaboración de materiales curriculares y también en el uso de los libros de texto. Se caracterizó a este primer factor como la ruptura del novel frente al realismo práctico del experto, ya que jóvenes y mayores se sitúan respectivamente en sus extremos.

El segundo factor (22.02\% de la varianza) agrupa, en su polo negativo, ítems que proponen una valoración positiva del uso de los libros de texto con opciones de respuesta siempre extremas., mientras que el polo positivo repite los ítems, pero con preferencia por opciones de respuesta moderadas, es por ello que se caracterizó al factor como "defensa a ultranza del libro de texto frente a la duda permanente en su uso".

Por último, el tercer factor (15.055\%), tiene un marcado carácter metodológico que enfrenta las ventajas de las metodologías más activas (polo negativo) frente a las de las más tradicionales (polo positivo).

Las proyecciones de los clústeres en los factores que se acaban de mencionar parecen encajar con una cierta lógica. En relación con el Factor 1, en el polo más idealista se sitúa, de forma extrema, el clúster 4 (los que abogan por el uso exclusivo de materiales de elaboración propia). El resto de clústeres se ubican, con un carácter moderado, en el polo generado por las ideas más prácticas.

Repitiendo el mismo proceso sobre el Factor 2 (Figuras 2 y 3) sitúan en el polo negativo en forma extrema, al clúster 3 (defensa del libro) y con un carácter más moderado al clúster 1 , por el contrario, en el polo positivo se encuentran los clústeres 2 y 4 . El primero de ellos de forma moderada y el segundo (partidarios del uso exclusivo de los materiales de elaboración propia) se instala justo en el centro del factor, parece como si los profesores pertenecientes a 
este clúster no opinaran sobre la utilidad o no del libro de texto.

Por lo que respecta al Factor 3, lo primero que se debe destacar es que los índices obtenidos por todos los clústeres son bastante bajos, es decir, no ocupan posiciones extremas en relación con la dimensión delimitada por este factor. Los clústeres 1 y 4 se sitúan en el polo configurado por las metodologías más activas, mientras que los clústeres 2 y 3 ocupan posiciones dentro del espacio de las metodologías de corte más tradicional.

Aunque la producción científica sobre la utilización de los libros de texto o de los materiales curriculares, de elaboración propia adaptados, es amplia, no lo es cuando se mezclan ambos componentes, que la producción científica suele tratar por separado. No obstante, la mayor demanda de formación para mejorar la capacidad de adaptar materiales curriculares a las exigencias específicas de los alumnos, que se ha detectado, se alinea con lo manifestado por Neuman, Pinkham y Kaefer (2015), quienes manifiestan exigencias explícitas en la necesidad de mejora de las capacidades de los enseñantes para la modificación y adaptación de los materiales. En la misma línea, se manifiestan Bismack, Arias, Davis y Palincsar (2015) que acentúan la importancia de los materiales curriculares elaborados por los propios docentes, como uno de los elementos de mayor influencia sobre el aprendizaje. Estos autores señalan también las carencias formativas de los docentes en este aspecto y manifiestan la necesidad de reformas de calado que favorezcan la posibilidad de producción docente. Por el contrario, cuando se seleccionan profesores competentes, las actividades de clase se organizan de forma más coherente (Iturra, 2015). Sostiene Givens (2000) que la posibilidad de elaboración de materiales adaptados es inestimable para el cambio curricular; su investigación pone de manifiesto que cuando a los profesores se les posibilita y proporcionan materiales adaptados disminuye el uso de los libros de texto. Cohen (2016) resalta el beneficio que se obtiene si se deja que los educadores sean agentes activos para la individualización del plan de estudios, así como la exigencia de una formación específica para esta labor docente.

La crítica al uso del libro de texto, señalada en el polo positivo del segundo factor y en el clúster número 4, no es una novedad en la producción científica. Así, Polikoff (2015) evidencia la distancia existente entre los contenidos desarrollados en los libros de texto, que inciden de forma particular en los procedimientos y en el uso de la memorización y las exigencias derivadas del currículo oficial. Del mismo modo, Thomas y Schmidt (2011) señalan las carencias de los libros de texto y defienden que, conforme los profesores van conociendo las necesidades de sus alumnos, es preferible que puedan escoger entre materiales educativos diversos, como sistema de adaptación a las necesidades detectadas. Por su parte, Travé et al. (2015) indica que un buen número de docentes se inclina por no usar el libro de texto, ya que considera que hay otros materiales alternativos, más adaptativos a las necesidades de los alumnos y critica que los manuales suelen estar dirigidos de forma prioritaria al logro de buenos resultados en pruebas estandarizadas.

La visión sobre la necesidad de complementar los libros de texto para mejorar su eficacia, que se encontró en el clúster 2 (profesorado ecléctico) y polo positivo del primer factor, queda también puesta de manifiesto en Harris et al. (2015). Estos autores complementaron las enseñanzas del libro de texto con una serie de materiales prácticos dirigidos a la enseñanza de la ciencia, así pudieron comprobar que los niveles de aprendizaje de los alumnos mejoraban cuando se usaban estos materiales complementarios.

Respecto a las bondades del uso de los materiales estandarizados, polo negativo del Factor 2 y clúster 3, la investigación no es tan exhaustiva como en los anteriores planteamientos; no obstante, se pueden rastrear algunos trabajos que asumen las bondades de su uso y buscan mejorar su eficacia, entre ellas se puede destacar a Watt (2016), que analiza el deseo del Gobierno australiano en la búsqueda de materiales estandarizados, señalando que los premios a la excelencia en la industria editorial educativa, establecidos 
por la Asociación Australiana de Editores, han conseguido aumentar la calidad de los materiales educativos. Negrín (2015), al analizar el proceso de adaptación a la acción educativa que requieren los profesores noveles, constata que la utilidad del libro de texto es alta, ya que configura la materia proporcionando un adecuado conocimiento didáctico, al tiempo que secuencia y jerarquiza de forma apropiada los contenidos.

Por último, se considera que las perspectivas de investigación se deberían encaminar, entre otros, hacia los aspectos siguientes: potenciar estudios para conocer la utilización real que el profesorado hace del libro de texto; fomentar indagaciones referidas a los procesos de elaboración de materiales curriculares por el profesorado y su relación con el grado de desarrollo profesional; indagar en la importancia que revisten los materiales curriculares en la formación inicial y permanente del profesorado y, por último, promover investigaciones que analicen las implicaciones del profesorado en procesos conjuntos de diseño, desarrollo y evaluación de materiales de aula y su difusión virtual para el marco educativo.

\section{Referencias}

Abdel Latif, M. M. M. (2012). Teaching a standard-based communicative English textbook series to secondary school students in Egypt: Investigating teachers' practices and beliefs. English Teaching: Practice and Critique, 11(3), 78-97. Recuperado de https://edlinked.soe.waikato.ac.nz/resea rch/files/etpc/files/2012v11n3art5.pdf

Benzécri, J. -P. (1976). L'analyse des données. Vol. 1. La taxinomie. Vol. 2. L'analyse des correspondances. París: Dunod.

Bismack, A. S., Arias, A. M., Davis, E. A., \& Palincsar, A. S. (2015). Examining student work for evidence of teacher uptake of educative curriculum materials. Journal of Research in Science Teaching, 52 (6), 816-846. https://doi.org/10.1002/tea.21220
Cañal, P., Travé, G., \& Pozuelos, F. (2011). Análisis de los obstáculos y dificultades de profesores y estudiantes en la utilización de enfoques de investigación escolar. Investigación en la Escuela, 73, 5-26. Recuperado de http://www.investigacionen laescuela.es/articulos/R73/R73.1.pdf

Chingos, M. M., \& Whitehurst, G. J. (2012). Choosing blindly: Instructional materials, teacher effectiveness, and the common core. Washington: Brookings Institution.

Cohen, E. H. (2016). Teacher autonomy within a flexible national curriculum: Development of Shoah (holocaust) education in Israeli state schools. Journal of Curriculum Studies, 48(2), 167-191. https://doi.org/10.1080/00 220272.2015.1033464

Givens, N. (2000). Curriculum materials as a vehicle for innovation: A case study of the nuffield design and technology project. Research in Science and Technological Education, 18(1), 71-83. Recuperado de htt ps://www.learntechlib.org/p/165302/

Harris, C. J., Penuel, W. R., D'Angelo, C. M., DeBarger, A. H., Gallagher, L. P., Kennedy, C. A., \& Krajcik, J. S. (2015). Impact of project-based curriculum materials on student learning in science: Results of a randomized controlled trial. Journal of Research in Science Teaching, 52(10), 1362-1385. https://doi.org/10.1002/tea.212 63

Huizinga, T., Handelzalts, A., Nieveen, N., \& Voogt, J.M. (2014). Teacher involvement in curriculum design: Need for support to enhance teachers' design expertise. Journal of Curriculum Studies, 46(1), 33-57. https:// doi.org/10.1080/00220272.2013.834077

Iturra, C. (2015). La organización de la enseñanza de la lectura en las aulas chilenas. Diferencias entre profesores calificados como básicos o como competentes. Universitas Psychologica, 14(2), 535-548. https://doi.org/10.11144/Ja veriana.upsy14-2.oela

Lebart, L., Morineau, A., \& Warwick, K. M. (1984). Multivariate descriptive statistical analysis: correspondence analysis and related 
techniques for large matrices. New York: Wiley.

Mansour, N. (2010). Impact of the knowledge and beliefs of Egyptian science teachers in integrating a STS Based Curriculum: A sociocultural perspective. Journal of Science Teacher Education, 21(5), 513-534. Recuperado de http://www.jstor.org/stable/ 43156564

Negrín, M. G. (2015). Políticas de formación, profesorado novel y libros de texto en el Sistema Educativo Argentino: una investigación sobre la enseñanza de la lengua y la literatura en la educación secundaria (Tesis doctoral inédita). Universidad de Granada, Granada.

Neuman, S. B., Pinkham, A., \& Kaefer, T. (2015). Supporting vocabulary teaching and learning in prekindergarten: The role of educative curriculum materials. Early Education and Development, 26(7), 988-1011. https://doi.org/10.1080/1040928 9.2015.1004517

Pilitsis, V., \& Duncan, R. (2012). Changes in belief orientations of preservice teachers and their relation to inquiry activities. Journal of Science Teacher Education 23, 8, 909-936. Recuperado de https://www.jstor. org/stable/43156682

Pingel, F. (2010). Guide Unesco pour l'analyse et révision des manuels scolaires. París: Institut Georg Eckert/Unesco.

Polat, N. (2010). Pedagogical treatment and change in preservice teacher beliefs: An experimental study. International Journal of Educational Research, 49(6), 195-209. https ://doi.org/10.1016/j.ijer.2011.02.003

Polikoff, M. S. (2015). How well aligned are textbooks to the common core standards in mathematics? American Educational Research Journal, 52 (6), 1185-1211. https:// doi.org/10.3102/0002831215584435

Rodríguez, J., \& Álvarez, D. (2017). A investigação sobre manuais escolares e materiais curriculares. Revista Lusófona de Educação, 36, 9-23. https://doi.org/10.2414 0/issn.1645-7250.rle36.01
Schizas, D., Papatheodorou, E., \& Stamou, G. (2018). Transforming "ecosystem" from a scientific concept into a teachable topic, philosophy and history of ecology informs science textbook analysis. Research in Science Education, 48(2), 267-300. https: //doi.org/10.1007/s11165-016-9568-0

Solís, E., Porlán, R., Rivero, A., \& Martín, R. (2012). Las concepciones de los profesores de ciencias de secundaria en formación inicial sobre metodología de enseñanza. Revista Española de Pedagogía, 70(253), 495-514. Recuperado de https://revistadepedagogia.org/wp-conte nt/uploads/2012/09/253-06.pdf

Stenhouse, L. (1997) Cultura y educación. Sevilla: Movimiento Cooperativo de Escuela Popular.

Thomas, P. L., \& Schmidt, R. (2011). Challenging texts: Challenging textbooks: Servants, not masters of our classrooms. The English Journal, 100(3), 91-96. Recuperado de https://www.jstor.org/stable 125790067

Travé, G., Pozuelos, F. J., \& Soto, A. (2015). Profesores y materiales curriculares en la enseñanza de la realidad social y natural. Análisis de concepciones sobre la práctica reflexionada del profesorado andaluz (España). Archivos Analíticos de Políticas Educativas, 23(56), 1-36. https://do i.org/10.14507/epaa.v23.1910

Watt, M. (septiembre, 2016). Aligning curriculum materials with the Australian curriculum: What is happening in the field and what needs to be done? Trabajo presentado en la Australian Curriculum Studies Association Biennial Conference, Adelaide, South Australia. (Número de servicio de reproducción de documentos ERIC ED 564 585)

\section{Notas}

* Artículo de investigación. Este trabajo ha sido el resultado del Proyecto de Excelencia "¿Cómo se realiza la enseñanza sobre la realidad social y natural en las aulas de 
Educación Infantil y Primaria de Andalucía? Estudio de las estrategias didácticas y propuestas de mejora” (SEJ-5219) y del Proyecto I $+\mathrm{D}$ " ¿Cómo mejorar la enseñanza elemental sobre el medio?: análisis del currículo, los materiales y la práctica docente" (EDU2009-12760EDUC). 